\title{
Predicting the Origin of Outflow Tract Ventricular Arrhythmias Using Machine Learning Techniques Trained With Patient-Specific Electrophysiological Simulations
}

\author{
Ruben Doste ${ }^{1}$, Miguel Lozano ${ }^{2}$, Juan Francisco Gomez ${ }^{3}$, Alejandro Alcaine ${ }^{1}$, Lluis Mont ${ }^{4}$, Antonio \\ Berruezo $^{5}$, Oscar Camara ${ }^{1}$, Rafael Sebastian ${ }^{2}$ \\ ${ }^{1}$ Physense, Universitat Pompeu Fabra, Barcelona, Spain \\ ${ }^{2}$ CoMMLab, University of Valencia, Valencia, Spain \\ ${ }^{3}$ Center for Research and Innovation in Bioengineering Polytechnic University of Valencia, Valencia, \\ Spain \\ ${ }^{4}$ Department of Cardiology, Unitat de Fibril-lacio Auricular (UFA), Hospital Clinic, Universitat de \\ Barcelona, Barcelona, Spain \\ ${ }^{5}$ Heart Institute, Teknon Medical Center, Barcelona, Spain
}

\begin{abstract}
Identifying the site of origin (SOO) of outflow tract ventricular arrhythmias (OTVAs) is key to plan radiofrequency ablation procedures. Currently, electrophysiologist try to extract that information pre-operatively from the ECG, and intraoperatively from electroanatomical maps. In this work, we study the prediction of the SOO by using machine learning approaches trained with patientspecific electrophysiological simulations of subjects that suffer OTVA. We built patient-specific models for 11 patients with OTVA, including an enhance description of the myofiber orientation in the outflow tracts, and simulated the sequence of activation, the BSPM and ECG. Following, we triggered the arrhythmia from twelve different SOO. Simulations were in agreement with real ECGs, hence we used the simulated ECGs to train our machine learning algorithms and classify the different SOO. According to our results, V3 lead provides useful information for SOO localization. Obtained classification rates show that simulated ECGs can help to determine right ventricle versus left ventricle outflow tract origin.
\end{abstract}

\section{Introduction}

Outflow tract ventricular arrhythmias (OTVAs) are a type of tachycardia in which the site of origin (SOO) of the ectopic beat is located near the right ventricle or left ventricle outflow tracts (RVOT and LVOT, respectively). Radiofrequency ablation is the usual treatment and is usually performed guided by electroanatomical mapping (EAM) data. However, a determination of the SOO before the ablation procedure is necessary in order to plan the intervention, the risk evaluation and the catheter approach. In order to overcome this challenging step, clinicians have developed several algorithms based on electrocardiograms (ECG) [1,2], although their accuracy is still limited. In this work, we propose the use of electrophysiological simulations combined with machine learning techniques to help clinicians to determine the SOO preoperatively. A high number of ECGs will be simulated from the most typical OTVA SOO $[2,3]$ and used to train different machine learning algorithms. After that, real ECGs recorded from OTVA patients will be tested and classified.

\section{Material and Methods}

Simulations were run using the data of 11 patients patients with idiopathic OTVAs submitted for ablation procedure at Hospital Clínic de Barcelona. Patient-specific biventricular models were generated from computed tomography CT images and using a fiber RBM specific to the OTs [4]. A total of 12 electrophysiological simulations were carried out in each geometry by changing the SOO according clinical reports [3]. Resulting potentials were propagated to the torso surface where ECGs were calculated. Finally, after simulations were validated, simulated ECGs were compared with patient ECG and used to train machine learning algorithms. These algorithms were k-nearest neighbors (k-NN), random forest (RF), supervised vector machine (SVM) and the self organized maps (SOM). Additional ECGs from unseen OTVA patients were then classified between RVOT origin and LVOT origin by the algorithms. 


\subsection{Model generation}

The biventricular geometries were segmented from CT images using a semi-automatic procedure based on region growing (3D Slicer software). Surface triangular meshes were post-processed and subsequently meshed with hexahedra by MeshGems-hexa software (Distene S.A.S., Bruyeres-le-Chatel, France). Resulting meshes had an average of 3.5 million nodes and 4 million elements, with an average edge length of $0.38 \mathrm{~mm}$. Cardiomyocyte orientation was included in the model using the outflow tract extended rule based method (OT-RBM) [4]. This RBM creates detailed fiber orientation in specific regions such as the outflow tracts, RV and the interventricular septum according to histological information $[5,6]$. These regions play a key role in the OTVA simulations. and their orientation highly differ from the myocyte orientation in rest of the ventricle.

A 3D torso model developed in previous works [7-9], that includes the different (bones, lungs, liver, ventricles, blood pools of the ventricles, blood vessels and body contour) was used. The segmented ventricle of each patient was fitted into the torso model and meshed with tetrahedra by TetGen [10], obtaining eleven models.

\subsection{Electrophysiological Modeling}

All the myocardium was modelled using the human ventricular ionic model formulated by ten Tusscher [11]. Transmural heterogeneity was added by defining three different action potential models for three different myocardial layers; endocardium, mid-miocardium and epicardium. Electrical propagation in tissue was modelled using the monodomain formulation and solved using ELVIRA software [12]. Conduction velocity was obtained by tuning the longitudinal and traversal conductivities and considering axisymmetric anisotropy. Several simulations were performed on a tissue slab geometry $(20 \times 7 \times 3 \mathrm{~mm})$ composed of hexahedral elements with an edge length of $\sim 0.38 \mathrm{~mm}$. A longitudinal conductivity value of $0.24 \mathrm{~S} / \mathrm{m}$ and a anisotropic ratio of 0.19 resulted in a conduction velocity of $0.68 \mathrm{~m} / \mathrm{s}$ along the myofiber direction, which is consistent with the values obtained experimentally in different works.

Electric propagation in the torso was considered isotropic. The different conductivity values assigned to each organ were extracted from the literature $[8,13]$.

\subsection{Simulation experiments}

A total of 12 simulations were performed in each of the 11 patient-specific geometries. Before simulating the OTVA, all models were stabilized by simulating 4 beats at sinus rhythm with a basic cyclic length of $1000 \mathrm{~ms}$, and a

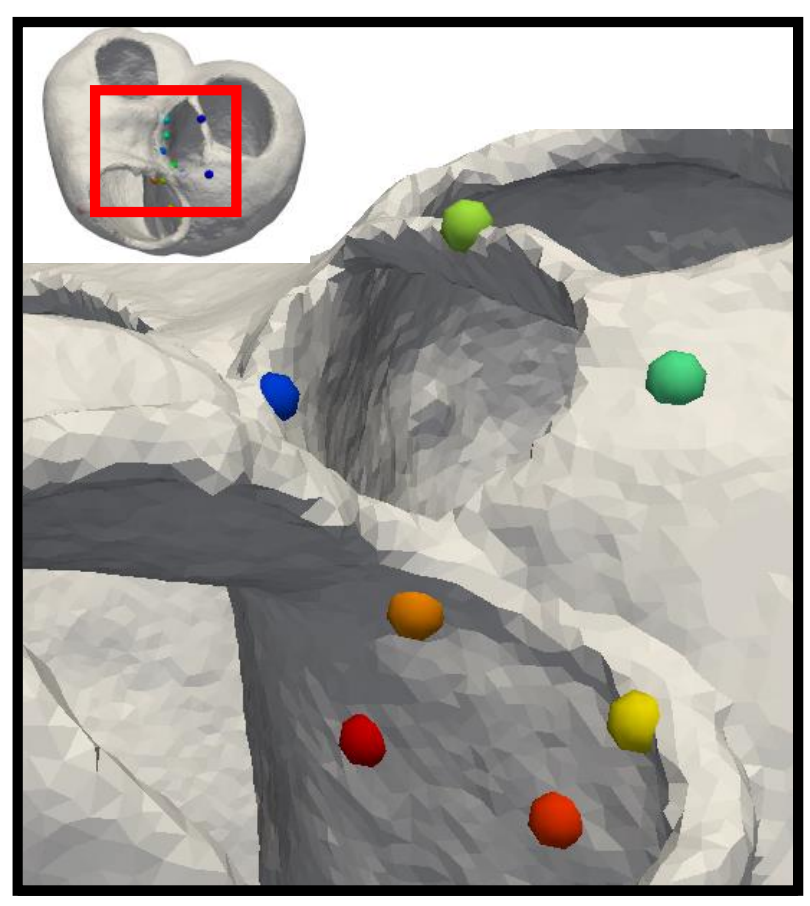

Figure 1. Location of the different points used as site of origin in the simulations.

time step of $0.02 \mathrm{~ms}$. Once the model was stabilized, we simulated the activation from the different ectopic focus, 7 SOO were placed in the LV and 5 in the RV. The location of the ectopic foci was chosen according to clinical observations $[2,3]$. An example of the SOO distribution in the simulations can be seen in Figure 1. After simulating the OTVAs at organ level (biventricular model), the resulting transmembrane potentials were used to computed the BSPM and ECGs. BSPMs were obtained by computing the extracellular potential at the torso surface nodes. ECGs were obtained by selecting the closest nine torso nodes to the standard 12-lead ECG electrode location.

\subsection{Signal Processing}

Simulated ECGs were compared with patient specific ECGs by calculating the correlation coefficient $(\rho)$ for each lead. We also defined the the 12 -lead $\rho$ by averaging the $\rho$ values over the 12 leads. This quantitative measure was already used in previous works for ECG comparison [14].

Signal processing was carried out using MATLAB software. All the simulated and patient ECGs were normalized in amplitude between -1 to 1 , where -1 and 1 were the minimum and maximum values of the 12-lead ECGs. Previous to calculating the 12-lead $\rho$, QRS complexes were manually extracted from the real patient ECGs and were aligned in each lead according with the highest value of the crosscorrelation. 
We performed a data augmentation by changing the torso lead configurations. A total of 13 different lead placements were chosen in order to add variability to the ECGs. We also calculated the LV/RV ratio in order to determine the LVOT vs RVOT origin. This ratio is calculated in each patient by dividing the mean of the $\rho$ values of all the LV SOO simulations by the mean of the $\rho$ values the RV SOO simulations. A value bigger than 1 will mean LVOT origin whereas a value minor than 1 will be characteristic of RVOT origin.

\subsection{Machine Learning Algorithms}

Different algorithms were used to classify test signals between RV and LV SOO. First, each ECG lead was classified between two classes (RVOT or LVOT origin) using self organizing maps (SOMs). Two SOM were trained independently with only simulated signals of one class. The best match of the two SOMs that had the smallest error respect to the input signal was set as the winner class. We also used RF, SVM and k-NN algorithms to classify the patient signals studying combinations of different ECG leads. Those models were previously trained only with simulated signals, and manually labeled to indicate the class.

\section{Results}

A total of 132 simulations of $300 \mathrm{~ms}$ were carried out. After data augmentation, $1716 \times 12$-lead ECGs were obtained. Comparison of the simulated ECGs and the patient ECGs presented good accordance. An example of the comparison between the patient ECG (V3 lead) and the simulated ECGs in a patient can be seen in Figure 2. The 12-lead $\rho$ calculated for each patient showed high values ( $\rho>0.9)$ in all the simulated signals that were close to the SOO. LV/RV ratio from the patients with origin in the LV showed higher values than those from the RV (1.07 vs 0.93 $\mathrm{p}<0.05$ for 12 -lead $\rho$ ). Only one patient presented $\rho$ values under 0.87 and bad classification. Simulated signals from this patient were excluded from the ML training.

ML training was performed using 1560 simulated 12lead ECG signals and the classification test using $35 \times$ 12-lead ECG signals from OTVA patients that underwent ablation in different SOO. Table 1 shows the accuracy obtained for each individual lead using SOM classification. The information obtained from the SOM classification was used to build predictors using several ECG leads. Table 2 shows the total accuracy obtained using different combinations of 12-lead ECGs. Selecting properly the signals (V2V5) increased substantially the performance of the SVM and k-NN classifiers.

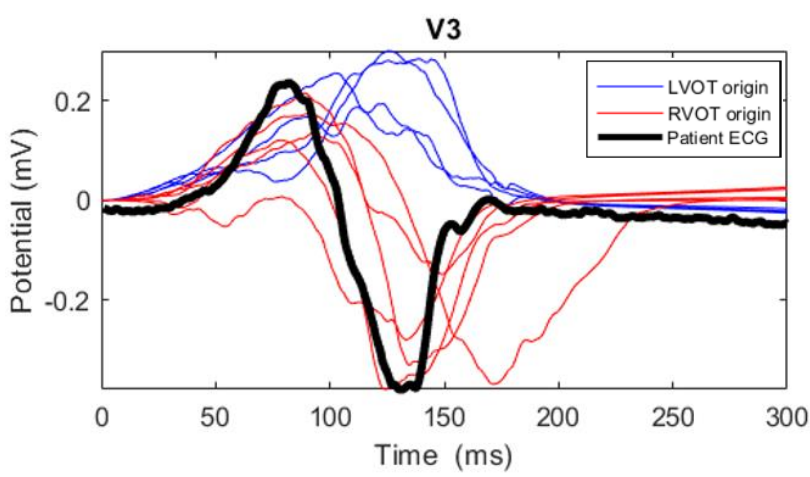

Figure 2. Simulated ECGs (V3 lead) in a RVOTA patient geometry. ECGs with a LVOT origin are pictured in blue whereas RVOT ECGs are in red. The black line corresponds to the real ECG of the RVOT patient.

Table 1. Accuracy in the real ECG signal classification using SOM in each lead of the ECG.

\begin{tabular}{ccc}
\hline \hline Precordial & Simulated $(\mathrm{n}=1560)$ & Real $(\mathrm{n}=35)$ \\
\hline I & 0.95 & 0.49 \\
II & 0.99 & 0.49 \\
III & 0.92 & 0.43 \\
aVR & 0.96 & 0.57 \\
aVL & 0.9 & 0.56 \\
aVF & 0.95 & 0.58 \\
V1 & 0.97 & 0.47 \\
V2 & 0.95 & 0.67 \\
V3 & 0.93 & 0.79 \\
V4 & 0.97 & 0.71 \\
V5 & 0.94 & 0.6 \\
V6 & 0.93 & 0.39 \\
\hline \hline
\end{tabular}

\section{Discussion}

In the present work, we have developed a pipeline for simulating OTVAs and show the potential role of the simulated ECG for training classification ML algorithms. Simulated ECG were first validated with patient ECGs, showing a good correspondence in 10 out of 11 patients. After validating the simulations, k-NN, SVM, RF and SOM algorithms were trained with the simulated data and used to classify simulated and real ECGs. As expected, classification of simulated data has a good performance (accuracy $>0.9$ ). On the other hand, results of using SOM to classify each lead of the real ECG confirm clinical observations: V2, V3 and V4 leads are the most impor- 
Table 2. Accuracy in the ECG signal classification using different combinations of ECG leads

\begin{tabular}{cccc}
\hline \hline & RF & SVM & k-NN \\
\hline 12 leads & 0.77 & 0.54 & 0.74 \\
Precordials & 0.63 & 0.43 & 0.63 \\
V2,V3,V4,V5 & 0.49 & 0.60 & 0.89 \\
\hline \hline
\end{tabular}

tant ones for OTVA SOO determination. Using all 12leads seems to add some noise to the classifier, since some leads do not provide consistent information with respect to the location of the SOO, and therefore worsen the performance. However, accuracy increases significantly by doing combinations of different leads. These results fit with electrophysiologist observations, that study the behaviour of a few selected ECG leads to determine the SOO. The main limitations of this work are the low number of patient ECG data available for testing the algorithms and the need of more OTVA simulations in more patients geometries. Simulation results and the obtained classification rates show that simulated ECGs can help to determine RVOT versus LVOT origin in OTVAs.

\section{References}

[1] Anderson RD, Kumar S, Parameswaran R, Wong G, Voskoboinik A, Sugumar H, Watts T, Sparks PB, Morton JB, McLellan A, Kistler PM, Kalman J, Lee G. Differentiating Right- and Left-Sided Outflow Tract Ventricular Arrhythmias. Circulation Arrhythmia and electrophysiology 2019;12(6):e007392.

[2] Lerman BB. Mechanism, Diagnosis, and Treatment of Outflow Tract Tachycardia. Nature Reviews Cardiology 2015; 12(10):597-608.

[3] Kamakura S, Shimizu W, Matsuo K, Taguchi A, Suyama K, Kurita T, Aihara N, Ohe T, Shimomura K. Localization of Optimal Ablation Site of Idiopathic Ventricular Tachycardia from Right and Left Ventricular Outflow Tract by Body Surface ECG. Circulation 1998;5-7.

[4] Doste R, Soto-Iglesias D, Bernardino G, Alcaine A, Sebastian R, Giffard-Roisin S, Sermesant M, Berruezo A, Sanchez-Quintana D, Camara O. A Rule-Based Method to Model Myocardial Fiber Orientation in Cardiac Biventricular Geometries With Outflow Tracts. International Journal for Numerical Methods in Biomedical Engineering 4 2019; 35(4):e3185.
[5] Sanchez-Quintana D, Doblado-calatrava M, Cabrera JA, Macías Y, Saremi F. Anatomical Basis for the Cardiac Interventional Electrophysiologist. BioMed Research International Ao 2015;2015.

[6] Greenbaum Ra, Ho SY, Gibson DG, Becker aE, Anderson RH. Left Ventricular Fibre Architecture in Man. British Heart Journal 1981;45(July 1980):248-263.

[7] Lopez-Perez A, Sebastian R, Izquierdo M, Ruiz R, Bishop M, Ferrero JM. Personalized Cardiac Computational Models: From Clinical Data to Simulation of Infarct-Related Ventricular Tachycardia. Frontiers in Physiology 2019; 10(May):580.

[8] Ferrer A, Sebastián R, Sánchez-Quintana D, Rodríguez JF, Godoy EJ, Martínez L, Saiz J. Detailed Anatomical and Electrophysiological Models of Human Atria and Torso for the Simulation of Atrial Activation. Plos One 2015; 10(11):e0141573.

[9] Godoy EJ, Lozano M, García-Fernández I, Ferrer-Albero A, MacLeod R, Saiz J, Sebastian R. Atrial Fibrosis Hampers Non-invasive Localization of Atrial Ectopic Foci From Multi-Electrode Signals: A 3D Simulation Study. Frontiers in Physiology 2018;9(MAY).

[10] Si H. TetGen, a Quality Tetrahedral Mesh Generator. AMC Transactions on Mathematical Software 2015;41(2):11.

[11] ten Tusscher K, Noble D, Noble PJ, Panfilov AV. A Model for Human Ventricular Tissue. AJP Heart and Circulatory Physiology 2004;286(4):H1573-H1589.

[12] Heidenreich EA, Ferrero JM, Doblaré M, Rodríguez JF. Adaptive Macro Finite Elements for the Numerical Solution of Monodomain Equations in Cardiac Electrophysiology. Annals of Biomedical Engineering 3 2010;38(7):23312345.

[13] MacLeod RS, Johnson CR, Ershler PR. Construction of an Inhomogeneous Model of the Human Torso for Use in Computational Electrocardiography. Proceedings of the Annual Conference on Engineering in Medicine and Biology 1991;13(2):688-689.

[14] Okada JI, Sasaki T, Washio T, Yamashita H, Kariya T, Imai Y, Nakagawa M, Kadooka Y, Nagai R, Hisada T, Sugiura S. Patient Specific Simulation of Body Surface ECG using the Finite Element Method. PACE Pacing and Clinical Electrophysiology 2013;36(3):309-321.

Address for correspondence:

Ruben Doste

Physense, Universitat Pompeu Fabra, Barcelona, Spain

ruben.doste@upf.edu 